\title{
New strains of norovirus and the mystery of viral gastroenteritis epidemics
}

\author{
Bonita E. Lee MD MSc, Xiao-Li Pang PhD
}

$\mathrm{N}$ orovirus was first identified in 1972 as the cause of a gastroenteritis outbreak in Norwalk, Ohio, and it became famous because of severe outbreaks on cruise ships in 2002. Norovirus is the cause of $50 \%-70 \%$ of gastroenteritis outbreaks and at least 5 global gastroenteritis epidemics since 1996. ${ }^{1}$ A biennial pattern of alternating quiescent and epidemic outbreak activity associated with the emergence of new norovirus variants has been identified in Alberta and some European countries. ${ }^{1,2} \mathrm{~A}$ new strain called GII.4 Sydney was first reported in Australia in March 2012 and has become the predominant strain worldwide. ${ }^{3}$ The GII.4 Sydney strain was first detected in Alberta, Canada, in September 2011, Denmark in January 2012, and the United States in September 2012. GII.4 Sydney has been the predominant strain worldwide since November 2012. ${ }^{4}$

Previously known as Norwalk-like virus, norovirus is a small nonenveloped positive-strand RNA virus that belongs to the Caliciviridae family. The receptor site and most of the genetic variability resides in the major capsid protein (VP1). ${ }^{5}$ Norovirus is classified into 5 groups (GI-GV) based on variations in VP1. Human noroviruses belong to GI, GII and GIV, with most infections caused by GI ( 8 genotypes) and GII (at least 19 genotypes). Up to $85 \%$ of global norovirus gastroenteritis epidemics and institutional outbreaks have been caused by various GII.4 variants since the 1990s, with GII.4 2012 Sydney as the most recent predominant strain.

There is no in vitro cell-culture system or in vivo small-animal model for human norovirus, which creates major challenges in the study of this pathogen. However, research using recombinant virus-like particles created by the in vitro expression of norovirus capsid proteins has suggested potential mechanisms that account for the epidemiology of the norovirus strains responsible for outbreaks. In the first potential mechanism, viruslike particles bind to human histo-blood group antigens, which are heterogeneous and complex carbohydrates found on red blood cells and mucosal epithelium of intestinal tract and other tissues and in body fluids including saliva. The expression of $\mathrm{ABH}$ histo-blood group antigens in tissue and body fluids other than red blood cells is regulated by the FTU2 secretor gene. The expression of the Lewis antigens depends on the combined phenotype of the FTU2 and Le gene. About $80 \%$ of the population express $\mathrm{A}, \mathrm{B}$ or $\mathrm{O}_{\mathrm{h}}$ on their tissues and histo-blood group antigens in their body fluids (secretors) corresponding to their inherited pattern of glycosyltransferase. People who do not express $\mathrm{ABO}$ antigens in tissue and body fluids (nonsecretors) still have ABO histoblood group antigens, produced by a different gene (FTU1), on their red blood cells. Challenge studies with a prototype Norwalk virus showed differences in susceptibility to infection depending on $\mathrm{ABO}$ types in secretors and a lower risk for infection among nonsecretors irrespective of their ABO type. An in vitro binding experiment showed that virus-like particles of various GI and GII genotypes recognized different $\mathrm{ABO}$ and Lewis antigens but that the virus-like particles of GII-4 variants from 1998 to 2008 showed broad binding to saliva from all $\mathrm{A}, \mathrm{B}$ and $\mathrm{O}$ secretors. ${ }^{6}$ The GII.4 variant was recently shown to infect secretors of all ABO blood groups, ${ }^{7}$ which could be a mechanism for their predominance.

The second proposed mechanism is that protective herd immunity drives the emergence of GII.4 variants every 2-4 years; this is supported by a study of epitope changes among GII.4 variants. ${ }^{8}$ Observed differences in binding to histoblood group antigen by different GII.4 variants suggest that the many distinct but similar antigens in the population supports the propagation of norovirus strains as they mutate under the selective pressure of host immunity, until the emergence of a predominant strain.

The third proposed mechanism is that the GII.4

\section{- Ker POINTS}

- The GII.4 Sydney strain is the latest norovirus variant to cause gastroenteritis epidemics worldwide.

- Diverse genetic and antigenic variability of norovirus may explain the periodic emergence and dominance of new strains.

- Our understanding of norovirus is limited by a lack of in vitro or in vivo culture systems.

- Vigilant molecular surveillance is key to preventing future norovirus epidemics and developing vaccines.

- Phase I clinical studies for norovirus vaccines have been published. 
norovirus genotype has a higher mutation rate and a faster pace of evolution than other genotypes. ${ }^{5} \mathrm{~A}$ virus with better ability to evolve will have higher overall epidemiologic fitness. Differences in the amino acid sequence of the capsid protein between GII.4 Sydney and the preceding epidemic strain (GII.4 New Orleans, 2010) ${ }^{4}$ might represent changes in antigenic characteristics or receptor binding of GII.4 Sydney that enabled it to emerge as the dominant global variant. There is no data suggesting that GII.4 Sydney causes more severe illness than other GII.4 variants.

A systemic review of published norovirus outbreaks reported an overall hospital admission rate of $0.5 \%$ and a mortality rate of $0.06 \%$, with higher mortality during outbreaks that occurred at health care facilities. ${ }^{9}$ Management of norovirus outbreaks is costly. Norovirus is also increasingly recognized as an important cause of sporadic gastroenteritis, but the disease burden is largely unknown because molecular diagnostic tests are not routinely available. A systematic review of studies in both developed and developing countries estimated that norovirus accounted for $10 \%$ $15 \%$ of severe gastroenteritis in children aged less than 5 years and $9 \%-15 \%$ of mild and moderate diarrhea among people of all ages..$^{10}$

The average incubation period is $24-48$ hours, and symptoms include vomiting ( $\geq 50 \%$ of cases), diarrhea, nausea, abdominal cramps, malaise and low-grade fever. Illness usually resolves in 12-72 hours but can last longer in young children, elderly people and those in hospital or with compromised immune systems. Asymptomatic infections with viral shedding have been documented. Several factors contribute to its high communicability (Box 1).

There are still unanswered questions about the management of norovirus outbreaks. The guidelines by the US Centers for Disease Control and Prevention for the prevention and control of

\section{Box 1: Factors that contribute to the high communicability of norovirus}

- The infectious dose is very low (18-100 particles), and patients of all ages are susceptible.

- High levels of viral shedding $\left(>10^{9}\right.$ particles $/ \mathrm{mL}$ stool in the first days after infection) can precede illness onset. Shedding gradually decreases over days to weeks but can last for months in patients who are immunocompromised.

- The virus is stable for a long time in the environment at temperatures from $0^{\circ} \mathrm{C}$ to $60^{\circ} \mathrm{C}$.

- The virus is dispersed by projectile vomiting.

- There are many modes of transmission (e.g., person-to-person contact, droplets, contaminated fomites, environment, food, water).

- Norovirus has a high degree of genetic and antigenic diversity because of mutations and recombination.

- Infection results in short-term immunity with incomplete cross-protection among strains. Repeat infections can occur throughout life, creating a large human reservoir. norovirus outbreaks were updated in 2011, but evidence is lacking for many recommendations. ${ }^{10}$ For example, the use of alcohol-based hand hygiene against norovirus is controversial because only surrogate animal caliciviruses have been studied in hand hygiene and disinfection protocols because human noroviruses are noncultivable. Although proper hand-washing with soap and running water is the most effective way to reduce norovirus contamination, alcohol-based hand sanitation is still recommended by the World Health Organization because it is better than no hand hygiene. Another area that requires more study is the role in secondary transmission of asymptomatic people who shed norovirus. There is no antiviral treatment for norovirus, and norovirus vaccines are under development. ${ }^{11}$

In temperate climates, new GII.4 variants usually emerge in the summer, leading to epidemic outbreaks the following winter. Vigilant surveillance of circulating norovirus strains is critical for the anticipation of novel variants, preparedness for upcoming epidemics, and development and production of vaccines.

\section{References}

1. Siebenga JJ, Vennema H, Zheng DP, et al. Norovirus illness is a global problem: emergence and spread of norovirus GII.4 variants, 2001-2007. J Infect Dis 2009;200:802-12.

2. Pang XL, Preiksaitis JK, Wong S, et al. Influence of novel norovirus GII.4 variants on gastroenteritis outbreak dynamics in Alberta and the Northern Territories, Canada between 2000 and 2008. PLoS One 2010;5:e11599.

3. van Beek J, Ambert-Balay K, Botteldoorn N, et al. Indications for worldwide increased norovirus activity associated with emergence of a new variant of genotype II.4, late 2012. Euro Surveill 2013;18:8-9.

4. Hasing ME, Lee BE, Preiksaitis JK, et al. Emergence of a new norovirus GII.4 variant and changes in the historical biennial pattern of norovirus outbreak activity in Alberta, Canada, from 2008 to 2013. J Clin Microbiol 2013;51:2204-11.

5. Bull RA, Eden JS, Rawlinson WD, et al. Rapid evolution of pandemic noroviruses of the GII.4 lineage. PLoS Pathog 2010; 6:e1000831.

6. Yang Y, Xia M, Tan M, et al. Genetic and phenotypic characterization of GII-4 noroviruses that circulated during 1987 to 2008. J Virol 2010;84:9595-607.

7. Frenck R, Bernstein DI, Xia M, et al. Predicting susceptibility to norovirus GII.4 by use of a challenge model involving humans. J Infect Dis 2012;206:1386-93.

8. Lindesmith LC, Costantini V, Swanstrom J, et al. Emergence of a norovirus GII.4 strain correlates with changes in evolving blockade epitopes. J Virol 2013;87:2803-13.

9. Desai R, Hembree CD, Handel A, et al. Severe outcomes are associated with genogroup 2 genotype 4 norovirus outbreaks: a systematic literature review. Clin Infect Dis 2012;55:189-93.

10. Updated norovirus outbreak management and disease prevention guidelines. MMWR Recomm Rep 2011;60:1-18. Available: www .cdc.gov/mmwr/pdf/rr/rr6003.pdf (accessed 2013 Mar. 10).

11. Atmar RL, Bernstein DI, Harro CD, et al. Norovirus vaccine against experimental human Norwalk Virus illness. $N$ Engl J Med 2011;365:2178-87.

Affiliations: Department of Pediatrics (Lee) and Department of Laboratory Medicine and Pathology (Pang), University of Alberta, Edmonton, Alta.

Contributors: Bonita Lee wrote the initial draft, and both authors revised the manuscript for important intellectual content. Both of the authors approved the final version submitted for publication. 